\title{
Economic and emission dispatch using cuckoo search algorithm
}

\author{
Rachid Habachi' ${ }^{1}$, Achraf Touil ${ }^{2}$, Abdellah Boulal ${ }^{3}$, Abdelkabir Charkaoui ${ }^{4}$, Abdelwahed Echchatbi $^{5}$ \\ Laboratory of Mechanical Engineering, Industrial Management and Innovation, \\ The Faculty of Sciences and Technology, Hassan 1st University, Morocco
}

\begin{tabular}{l}
\hline \hline Article Info \\
\hline Article history: \\
Received Feb 14, 2019 \\
Revised Apr 4, 2019 \\
Accepted Apr 4, 2019 \\
\hline Keywords: \\
Cuckoo searchalgorithm \\
Economic dispatch problem \\
Emission cost \\
Fuel cost \\
Smart grid \\
\hline
\end{tabular}

\begin{abstract}
The economic dispatch problem of power plays a very important role in the exploitation of electro-energy systems to judiciously distribute power generated by all plants. This paper proposes the use of Cuckoo Search Algorithm (CSA) for solving the economic and Emission dispatch. The effectiveness of the proposed approach has been tested on 3 generator system.CSA is a new meta-heuristic optimization method inspired from the obligate brood parasitism of some cuckoo species by laying their eggs in the nests of other host birds of other species. The results shows that performance of the proposed approach reveal the efficiently and robustness when compared results of other optimization algorithms reported in literature.
\end{abstract}

Copyright $\odot$ 2019Institute of Advanced Engineering and Science. All rights reserved.

\section{Corresponding Author:}

Rachid Habachi,

Laboratory of Mechanical Engineering, Industrial Management and Innovation,

Faculty of Sciences and Technology,

Hassan 1st University,

PO Box 577, Settat, Morocco.

Email: habachidepartementgegm@gmail.com

\section{INTRODUCTION}

Smart grids are a set of technologies, concepts and approaches, allowing the integration the generation, transmission, distribution and use into one internet by full use of advanced sensor measurement technology, communications technology, information technology, computer technology, control technology, new energy technologies [1]. However, Smart Grid uses digital technology to control grid and choosing the best mode of power distribution to reduce energy consumption, reduce costs, increase reliability and also increase transparency in the network. Therefore, the system intelligent will have a significant impact in the fields of finance and economics of the power industry [2]. Although, The traditional network is a one-way network in which the electrical energy produced in power plants is channeled to consumers without information to create an automated and distributed network of advanced power supplies.

EDP is also applied in the integrated system for scheduling power plants. A few methods have been published to solve the ED problem and Optimal Power Flow (OPF). Researchers have published a few methods to solve ED and OPF problems. Direct method is accurate and very simple but limited by the quadratic objective function [3]. The economic dispatch (ED) is one of the power management tools that is used to determine real power output of thermal generating units to meet required load demand. The ED results in minimum fuel generation cost, minimum transmission power loss while satisfying all units, as well as system constraints [4-5].

The demand for electricity is increasing in a large factor in today ${ }^{e e}$ life, which makes it highly crucial to run generators at very minimal cost. This is the main factor of an Economic dispatch problem. With the unexceptional production of carbon emissions in thermal power plant, its needed to optimize the emission together with the optimization of cost which acts as two vital parts of Economic dispatch problem. The economic dispatch solution provides the best minimum cost of fuel and emission. This indirectly makes 
lower cost for electricity and makes electrical utilities more competitive in the market. As the energy cannot be stored, it requires highly efficient estimation scenarios including transmission and distribution systems to make the same work effectively.

Various technologies have been introduced to solve the optimization of Economic Load Dispatch problems. The selection of the optimization algorithm is the important part of the problem involving economic dispatch. The EDP is developed based on real-valued codification. In modern methodology only the cost function is evaluated and a global minimum solution is computed, independently of the cost function. The use of digital computers for obtaining loading schedules were investigated and used today. The ED is a static problem is to say we must define at a given powers generated by each power plant to power a load as economically as possible. To solve this problem the optimization methods are used.

Conventional optimization techniques [6,7]. have long been applied to solve the ED problem such as Quadratic Programming [8,9]. linear programming [10] sequential approach with a matrix framework (SAMF),[11]. modified Lambda-iteration method [12], Newton Raphson and Lagrangian multiplier (LM) algorithms [13], In the real-design cases, the number of decision variables (i.e. power units) of the ED area are very large. The objective criterion to be minimized could also have too many local minimum which might not lead to the minimum cost and the best generation schedule of power system units. Therefore, efficient search algorithms are needed.

Many deterministic optimization approaches were proposed to solve the ELD problem, including lambda iteration method [11], gradient method, linear programming [12], non-linear programming, dynamic programming [13] and quadratic programming [14]. But these methods require enormous efforts in terms of computation. Due to complexities of computing, therefore efficient algorithm to find optimal solution like genetic algorithm [15, 16], particle swarm optimization [17], evolutionary programming, artificial bee colony optimization [18, 19], and biogeography based optimization; bacterial foraging and also their variants came into implement. Bio-inspired meta-heuristic algorithms have recently shown the efficiency in dealing with many nonlinear optimizations constrained problems for finding the optimal solution.

The remaining organization of this paper is as follows. Section 2 presents the problem formulation of the EDP. Handling of constraints and implementation of the proposed CSA to ED problem are addressed in Sect. 3. Section 4 reports results of the proposed CSA method. A number of case studies using standard test systems are used to test the proposed method. The comparisons of results between the proposed method and existing methods are also carried out in this section. The discussion is followed in Sect. 5. After all, the conclusion is given.

\section{PROBLEM FORMULATION}

The generating units are loaded economically such a way to reduce the operating cost. Considering the valve point effect the economic dispatch formulated the objective function as given below

$$
F_{i}\left(P_{i}\right)=a_{i}+b_{i} P_{i}+c_{i} P_{i}^{2}
$$

where

$a_{i}$, bi ,ci are the fuel cost coefficients of generator $i$

$\mathrm{P}_{\mathrm{i}}$ is the power generated by unit $\mathrm{Ii}, \mathrm{MW}$

$F_{i}\left(P_{i}\right)$ is the fuel cost function of unit $i$

The objective function of the ED problem is to minimize the total production cost, which be written as:

$$
F_{T}\left(P_{T}\right)=\sum_{i=1}^{N} F_{i}\left(P_{i}\right) \quad i=1,2, \ldots, N
$$

The new objective function by considering valve point loading along with total fuel cost becomes,

$$
F_{T}\left(P_{T}\right)=\sum_{i=1}^{N} a_{i}+b_{i} P_{i}+c_{i} P_{i}^{2}+(\operatorname{disin}(e i(P i m-P i)))
$$

where

$a_{i}, b_{\mathrm{i}}, \mathrm{c}_{\mathrm{i}} \mathrm{d}_{\mathrm{i}}$ and $\mathrm{e}_{\mathrm{i}}$ are the fuel cost coefficients of generator $\mathrm{i}$

$P_{i}$ is the power generated by unit i, MW

Piminis the minimum generartion limit of unit i,MW

$F_{T}\left(P_{T}\right)$ is the total fuel cost $\$ / \mathrm{hr}$

The solution of EDP can be highly improved by introducing higher order generator cost functions. Cubic cost function displays the actual response of thermal generatorsmore accurately. The cubic fuel cost function of a thermal generating unit is represented as follows [20]: 


$$
F_{i}\left(P_{i}\right)=a_{i}+b_{i} P_{i}+c_{i} P_{i}^{2}+d_{i} P_{i}^{3}
$$

In order to minimize the pollutants, emission is consiered along with economic dispatch. The generator can be modelled as having a quadratic relation between the amount of pollutants released and the power generated.The mathematical formulation for generator is given by,

$$
E i(P i)=\alpha i+\beta i P_{i}+\gamma i P_{i}^{2}
$$

where

$\alpha \mathrm{i}, \beta \mathrm{i}, \gamma \mathrm{i}$ are the emission coefficients of generator $\mathrm{i}$

$\mathrm{P}_{\mathrm{i}}$ is the power generated by unit $\mathrm{i}, \mathrm{MW}$

$\mathrm{Ei}(\mathrm{Pi})$ is the fuel cost function of unit $\mathrm{i}$

The total emission for the entire system of $\mathrm{N}$ generators can then be calculated as,

$$
E_{T}\left(P_{T}\right)=\sum_{i=1}^{N} E_{i}\left(P_{i}\right)=\sum_{i=1}^{N} \alpha i+\beta i P_{i}+\gamma i P_{i}^{2}
$$

The new Emission function becomes,

$$
\mathrm{E}_{\mathrm{T}}\left(\mathrm{P}_{\mathrm{T}}\right)=\sum_{\mathrm{i}=1}^{\mathrm{N}} \mathrm{E}_{\mathrm{i}}\left(\mathrm{P}_{\mathrm{i}}\right)=\sum_{\mathrm{i}=1}^{\mathrm{N}} \alpha \mathrm{i}+\beta \mathrm{iP}_{\mathrm{i}}+\gamma \mathrm{i} \mathrm{P}_{\mathrm{i}}^{2}+\varepsilon \mathrm{i} \exp (\delta \mathrm{i} \mathrm{Pi})
$$

where

$\alpha \mathrm{i}, \beta \mathrm{i}, \gamma \mathrm{i}, \varepsilon \mathrm{i}, \delta \mathrm{i}$,are the emission coefficients of generator $\mathrm{i}$

$P_{i}$ is the power generated by unit $i, M W$

$\mathrm{Ei}(\mathrm{Pi})$ is the fuel cost function of unit $\mathrm{i}$

$\mathrm{E}_{\mathrm{T}}\left(\mathrm{P}_{\mathrm{T}}\right)$ is the total emission, ton/hr.

$\mathrm{N}$ is the number of generating units

subject to real power balance equation. The total active power output of generating units must be equal to total power load demand plus power loss:

$$
\sum_{i=1}^{N} P i=P_{D}+P_{L}
$$

where the power loss PL is calculated by the below formulation [4]:

$$
P_{L}=\sum_{i=1}^{N} \sum_{j=1}^{N} P_{i} B_{i j} P_{j}+\sum_{i=1}^{N} B_{O i} P_{i}+B_{O O}
$$

Generator capacity limits The active power output of generating units must be within the allowed limits:

$$
P_{i . \min } \leq P_{i} \leq P_{i . \max }
$$

\section{CUCKOO SEARCH ALGORITHM (CSA)}

Cuckoo search (CS) is inspired by some species of a bird family called cuckoo because of their special lifestyle andaggressive reproduction strategy. This algorithm was proposed by Yang and Deb [21]. The CS is an optimization algorithm based on the brood parasitism of cuckoo species by laying their eggs in the communal nests ofother host birds, though they may remove others' eggs to increase the hatching probability of their own eggs. Some host birds do not behave friendly against intruders and engage in direct conflict with them. If a host bird discovers the eggs are nottheir own, it will either throw these foreign eggs away or simply abandon its nest and build a new nest elsewhere [22].

The Cuckoo search algorithm contains a population of nests or eggs. Each egg in a nest represents a solution and a cuckoo egg represents a new solution. If the cuckoo egg is very similar to the host's, then this cuckoo egg is less likely to be discovered; thus, the fitness should be related to the difference in solutions. The better new solution (cuckoo) is replaced with a solution which is not so good in the nest. In the simplest form, each nest has one egg. When generating new solutions for $x^{(t+1)}$, say cuckoo $i$, a Lévy flight is performed:

$$
x^{t+1}=x i^{t}+\alpha \oplus \operatorname{Levy}(\lambda)
$$


where $\alpha>0$ is the step size which should be related to the scales of the problem of interest. In most cases, wecan use $\alpha=\mathrm{O}$ (1). The product $\bigoplus$ means entry-wise multiplications. Lévy flights essentially provide a randomwalk while

$$
\operatorname{Levy}(\lambda)=u=t^{-\lambda}, 1 \leq \lambda \leq 3
$$

random walk process which obeys a power-lawstep-length distribution with a heavy tail. The rules for CSA are described as follows:

- Each cuckoo lays one egg at a time, and dumps it in arandomly chosen nest;

- The best nests with high quality of eggs (solutions)will carry over to the next generations;

- The number of available host nests is fixed, and ahost can discover a foreign egg with a probability pac[0, 1]. In this case, the host bird can either throwthe egg away or abandon the nest so as to build acompletely new nest in a new location.

The later assumption can be approximated by the fraction paof the $n$ nests which are replaced by new ones(with new random solutions). With these three rules, thebasic steps of the CS can be summarized as the pseudo-codeshownbellow,

1) Define the objective function $\operatorname{Td} \mathrm{f}(\mathrm{x}), \mathrm{x}=\left(\mathrm{x}_{1}, \mathrm{x}_{2}, \mathrm{x}_{3}, \ldots, \mathrm{x}_{\mathrm{d}}\right)^{\mathrm{T}}$

2) Set $n, p a$, and MaxGeneration parameters

3) Generate initial population of $n$ available nests

4) Move a cuckoo (i) randomly by Lévy flights

5) Evaluate the fitness fi

6) Randomly choose a nest (j) among $\mathrm{n}$ available nests

7) If fi $>$ fj the replace $\mathrm{j}$ by the new solution

8) Abandon a fraction pa of worse nests and create the same fraction of new nests at new location via Lévy flights

9) Keep the best solutions (or nests with quality solutions)

10) Sort the solutions and find the best current solution

11) If stopping criteria is not satisfied, increase generation number and go to step 4

12) Post-process results and find the best solution among all.

The pseudo code of CSA for EDP is shown in Figure 1.

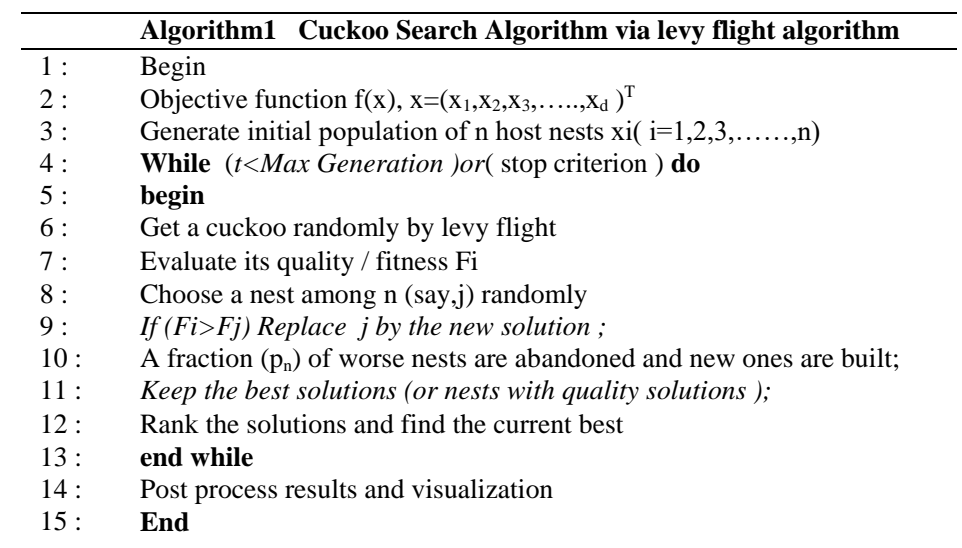

Figure 1. Pseudocode of Cuckoo search algorithm for EDP

\section{RESULTS AND ANALYSIS}

In this section, we present the results obtained based on Cuckoo Search Algorithm (CSA) for solving the economic and Emission dispatch and compare this results with the CM (Conventional Method) [23] and Particle Swarm Optimization [24]. A three units power unit system to explore our idea on using CSA to find the optimal set of power generation of the system. CSA will be used in this paper to solve the economic and Emission dispatch.The programs are developed in MATLAB 7.9 environment.

The adopted system is expected to produce demand power of $400 \mathrm{MW}$. The Table 1 shows the cost coefficient of the three generators, under study, while the matrix is the loss coefficient matrix of the three units power system..Generator emission coefficients for IEEE-30-bus system is provided in Table 2 given below. From the results of Table 3, we notice that CSA give us the same production cost, and CM gives a 
slightly lower cost of $\$ 0.5 / \mathrm{h}, \mathrm{CSA}$ gives us a good production cost and good accuracy,. Through the results found, we can say that there is a small difference between the optimal generated powers, the cost of production as well as the transmission losses between the different algorithms. The optimization of production cost is better when applying CSA compared to CM. This leads us to compare the results of CSA with those of PSO.The total cost given by PSO is $20813 \$ / \mathrm{h}$ and around $20812.5 \$ / \mathrm{h}$ for CSA, which represents a difference of $0.5 \$ / \mathrm{h}$. The transmission losses evaluated by the three approaches remained very close for the three algorithms respectively (PSO) 7.5681MW, (CM) 7.5687 MW, (CSA) 7.5681 MW.

In Figure 2, we show the convergence of the metaheuristic search process based on CSA in both the best and average cases. To see the difference between our new approach and another known method, we will compare the production cost found by CSA to that found by PSO [25]. The comparison is represented by the graph in Figure 3 and Figure4. It can be seen that CSA provided the minimum fuel cost and Emission cost in this case compared to other reported methods in the literature. This shows that the CSA is more effective in finding the best load for the three generator system.

In this case, we will test the operation of CSA. For this, we will use a simple network of 14 nodes with 3 production units. The total demand of the network is equal to $400 \mathrm{MW}$ and loss coefficients are as follows:

$$
B_{I J}=10^{-5}\left[\begin{array}{lll}
7.1 & 3.0 & 2.5 \\
3.0 & 6.9 & 3.2 \\
2.5 & 3.2 & 8.0
\end{array}\right]
$$

Table1. The parameters of the cost function and generators limits of the three -unit system

\begin{tabular}{cccccc}
\hline Units & $\mathrm{a}(\$ / \mathrm{MW})$ & $\mathrm{b}(\$ / \mathrm{MW})$ & $\mathrm{c}(\$)$ & Pmin $(\mathrm{MW})$ & Pmax $(\mathrm{MW})$ \\
\hline 1 & 0.03546 & 38.30553 & 1243.5311 & 35 & 210 \\
2 & 0.02111 & 36.32782 & 1658.5696 & 130 & 325 \\
3 & 0.01799 & 38.27041 & 1356.6592 & 125 & 315 \\
\hline
\end{tabular}

Table 2. Sample Emission Coefficients of the three -unit system

\begin{tabular}{cccccc}
\hline Units & $\alpha i$ & $\beta i$ & $\gamma i$ & $\varepsilon i$ & $\delta i$ \\
\hline 1 & 4.091 & -5.554 & 6.49 & $2.00 \mathrm{E}-03$ & 2.857 \\
2 & 2.543 & -6.047 & 5.638 & $5.00 \mathrm{E}-04$ & 3.333 \\
3 & 4.258 & -5.094 & 4.586 & $1.00 \mathrm{E}-06$ & 1 \\
\hline
\end{tabular}

Table 3. Results of the economic dispatching of three-unit system

\begin{tabular}{llll}
\hline & CM & PSO & CSA \\
\hline P1 (MW) & 82.0870 & 82.0786 & 82.0783 \\
P2 (MW) & 175.0042 & 175.0050 & 175.00 .48 \\
P3 (MW) & 150.4938 & 150.5002 & 150.4961 \\
P1 (MW) & 7.5687 & 7.5681 & 7.5681 \\
Fuel cost (\$/h) & 20813 & 20813 & 20812.5 \\
Total Emission(ton/hr) & 0.213921 & 0.213841 & 0.213841 \\
Total emissioncost $(\$ / h)$ & 330.018 & 329.018 & 328.7687 \\
\hline
\end{tabular}

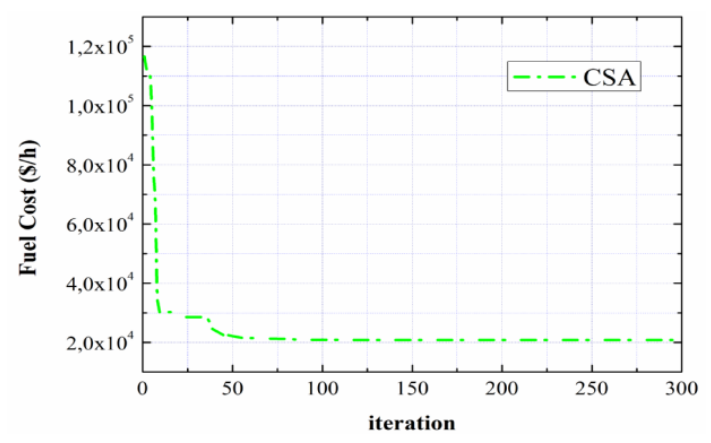

Figure 2. Cost convergence characteristic of 3-generator system 


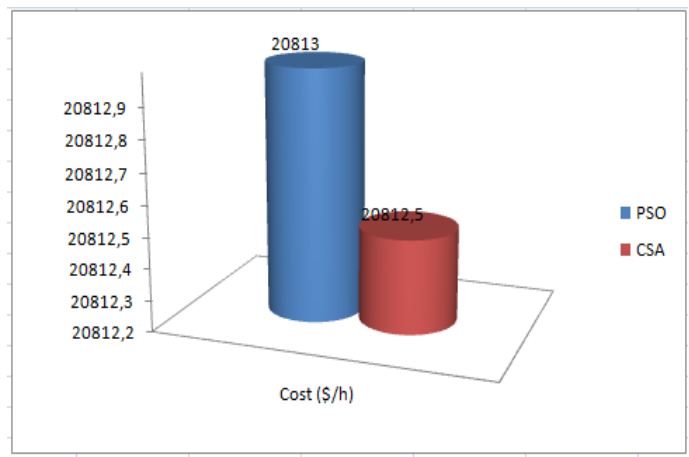

Figure 3. Comparison Fuel cost $(\$ / \mathrm{h})$ graph between CSA and PSO

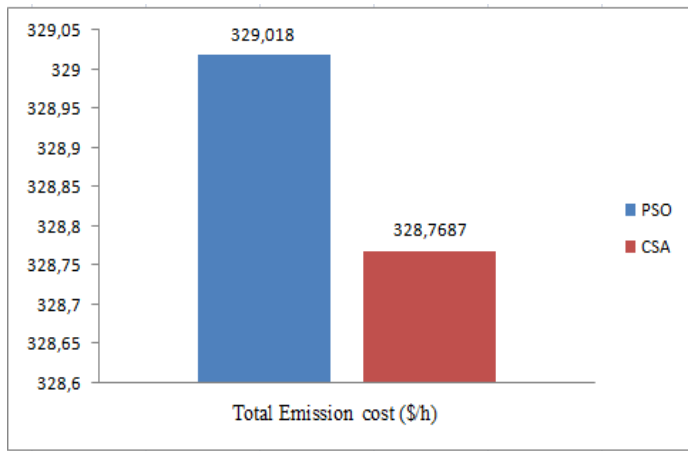

Figure 4. Comparison graph Total Emission cost $(\$ / h)$ Between CSA and PSO

\section{CONCLUSION}

In this paper, we proposed a Cuckoo search algorithm to solvethe economic and Emission dispatch . The practicality of the proposed metaheuristics CSA was tested for three power generators. The gained results were compared to existing results based on PSO and CM methods. It was shown that CSA are superior in obtaining a combination of power loads that fulfill the problem constraints and minimize the total fuel cost and emission cost . CSA found to be efficient in finding the optimal power generation loads. CSA was capable of handling the non-linearity of ED problem. The evolved power using CSA minimized both the cost of generated power, the total power loss in the transmission and maximizes the reliability of the power provided to the customers. The programs were developed using MATLAB and tested a network of 14 nodes. The results have shown that our CSA to give us a better performance with optimal results in all cases and respecting the constraints imposed.

\section{ACKNOWLEDGEMENTS}

The authors are very much thankful to the unanimous reviewers of the paper and editors of the journal for their constructive and helpful comments that improved the quality of the paper.

\section{REFERENCES}

[1] C.H. Rui and P. Xu, "Study on Smart Grid System Based on System Dynamics,"TELKOMNIKA Telecommunication Computing Electronics and Control,vol/issue: 12(12), pp. 7979-7986, Dec 2014.

[2] Shahinzadeh H. and HasanalizadehK. A.,"Implementation of Smart Metering Systems: Challenges and Solutions,"TELKOMNIKA Telecommunication Computing Electronics and Control, vol/issue: 12(7), 2014.

[3] Hermagasantos Z., et al. "Implementation of Electricity Competition Framework with Economic Dispatch Direct Method,'TELKOMNIKA Telecommunication Computing Electronics and Control,vol/issue: 10(4), pp. 667-674, 2012.

[4] Wollenberg B. and Wood A.,"Power generation, operation and control," New York, Wiley, 1996.

[5] Dieu V. N., et al.,"Pseudo-gradient based particle swarm optimization method for nonconvex economic dispatch,"in Power, control and optimization, Springer, New York, pp. 1-27, 2013.

[6] K. Aoki and T. Satoh, "New algorithms for classic economic load dispatch," IEEE Transactions on Power Apparatus and Systems, vol/issue: PAS-103(6), pp. 1423-1431, Jun 1984.

[7] J. K. Delson and S. M. Shahidehpour, "Linear programming applications to power system economics, planning and operations," IEEE Transactions on Power Systems, vol/issue: 7(3), pp. 1155-1163, Aug 1992.

[8] S. Subramanian and S. Ganesan, "A simple approach for emission constrained economic dispatch problems," International Journal of Computer Applications, vol/issue: 8(11), pp. 39-45, Oct 2010.

[9] D. D. Obioma and A. M. Izuchukwu, "Comparative analysis of techniques for economic dispatch of generated power with modified lambda-iteration method," in Proceedings of the 2013 IEEE International Conference on Emerging Sustainable Technologies for Power ICT in a Developing Society (NIGERCON), pp. 231-237, Nov 2013.

[10] S. K. Mishra and S. K. Mishra, "A comparative study of solution of economic load dispatch problem in power systems in the environmental perspective," Procedia Computer Science, vol. 48, pp. 96-100, 2015.

[11] S. K. Dewangan, et al., "A Traditional Approach to Solve Economic Load Dispatch Problem Considering the Generator Constraints,"IOSR Journal of Electrical and Electronics Engineering (IOSR-JEEE), vol/issue: 10(2), pp. 27-32, 2015.

[12] B. Stott, et al., "Power System Security Control Calculations Using Linear Programming,"Part I, IEEE Trans. on Power Apparatus and Systems, vol/issue: PAS-97(5), pp. 1713-1720, Sep 1978. 
[13] D. L.Travers and R. Kaye, "Dynamic dispatch by constructive dynamic programming,"IEEE Trans. on Power Systems, vol/issue: 13(1), pp. 72-78, Feb1998.

[14] Gaing, "Particle swarm optimization to solving the economic dispatch considering the generator constraints,"IEEE Trans. Power Syst., vol. 18, pp. 1187-1195, Aug 2003.

[15] E. Lin and G. L. Viviani, "Hierarchical Economic Dispatch for piecewise quadratic cost functions," IEEE Transactions on power apparatus and systems, vol/issue: PAS-103(6), pp. 1170-1175, 1984.

[16] G. Kumar and R. Singh, "Economic Dispatch of Power System Optimization with Power Generation Schedule Using Evolutionary Technique," International Journal of Advanced Research in Electrical Electronics and Instrumentation Engineering, vol/issue: 3(7), Jul 2014.

[17] K. S. Kumar, et al., "Economic load dispatch with emission constraints using various PSO algorithms," WSEAS Transactions on Power Systems, vol/issue: 3(9), pp. 598-607, 2008.

[18] H. Vennila andT. R. D. Prakash, "A solution for environmental constrained Economisc Dispatch Problems using Honey Bee Algorithm,"International journal of Computer Application, vol/issue: 47(22), pp. 13-17, 2012.

[19] H. VennilaandT. R. D. Prakash, "Particle Swam Optimisation Technique for solving Economic Dispatch Problems," Elsevier Journal of Procedia Engineering, vol. 48, pp. 2009-2021, 2012.

[20] Jiang A., et al." "Economic dispatch with non-monotonically increasing incremental cost units and transmission system losses,"Discussion. IEEE Trans Power Syst,vol. 10, pp. 891-897, 1995.

[21] X. S. Yang and S. Deb, "Cuckoo search via Levy flights," in Proceedings of world congress on nature \& biologically inspired computing,USA; IEEE Publications, pp. 210-214, Dec 2009.

[22] X. S. Yang and S. Deb, "Engineering optimization by cuckoo search," Int. J. Mathematical Modelling and Numerical Optimisation, vol/issue: 1(4), pp. 330-343, 2010.

[23] D. D. Obioma and A. M. Izuchukwu, "Comparative analysis of techniques for economic dispatch of generated power with modified lambda-iteration method," in Proceedings of the 2013 IEEE International Conference on Emerging Sustainable Technologies for Power ICT in a Developing Society (NIGERCON), pp. 231-237, Nov 2013.

[24] A. L. Devi and O. V. Krishna, "Combined economic and emission dispatch using evolutionary algorithms: A case study," ARPN Journal of Engineering and Applied Sciences, vol/issue: 3(6), pp. 28-35, 2008.

[25] Z. L. Gaing,"Particle Swarm Optimization to Solving the Economic Dispatch Considering the Generator Constraints,"IEEE transactions on power systems, vol/issue: 18(3), pp. 1187-1195, 2003. 